\title{
Quaternionic maps and minimal surfaces
}

\author{
JINGYI CHEN AND JIAYU LI
}

\begin{abstract}
Let $\left(M, J^{\alpha}, \alpha=1,2,3\right)$ and $\left(N, \mathcal{J}^{\alpha}, \alpha=1,2,3\right)$ be hyperkähler manifolds. We study stationary quaternionic maps between $M$ and $N$. We first show that if there are no holomorphic 2-spheres in the target then any sequence of stationary quaternionic maps with bounded energy subconverges to a stationary quaternionic map strongly in $W^{1,2}(M, N)$. We then find that certain tangent maps of quaternionic maps give rise to an interesting minimal 2-sphere. At last we construct a stationary quaternionic map with a codimension-3 singular set by using the embedded minimal $\mathbb{S}^{2}$ in the hyperkähler surface $\widetilde{M}_{2}^{0}$ studied by AtiyahHitchin.
\end{abstract}

Mathematics Subject Classification (2000): 53C26 (primary); 53C43, 58E12, 58E20 (secondary).

\section{Introduction}

A Riemannian manifold is called hyperkähler if it possesses covariant constant complex structures $I, J, K$ which satisfy the quaternionic relation

$$
I^{2}=J^{2}=K^{2}=I J K=- \text { identity. }
$$

Associated to $I, J, K$ there is a natural family of covariant constant complex structures $a I+b J+c K$ where $(a, b, c)$ is a unit vector in $\mathbb{R}^{3}$. A hyperkähler manifold is Ricci-flat with dimension $4 k$. Let $M$ and $N$ be two hyperkähler manifolds with complex structures $J^{\alpha}$ and $\mathcal{J}^{\beta}$ respectively for $\alpha, \beta=1,2,3$ which satisfy the quaternionic identities. A smooth map $f: M \rightarrow N$ is called a quaternionic map if

$$
\sum_{\alpha, \beta=1}^{3} A_{\alpha \beta} \mathcal{J}^{\beta} \circ d f \circ J^{\alpha}=d f
$$

where $A_{\alpha \beta}$ denote the entries of a constant matrix $A$ in $S O(3)$. Since $S O(3)$ preserves the quaternionic identities, we can always choose complex structures $J^{\alpha}$ for $M$ and $\mathcal{J}^{\beta}$ for $N$ such that $A_{\alpha \beta}=\delta_{\alpha \beta}$ in (1.1).

The first author is partially supported by NSERC and the second author is partially supported by NSFC.

Pervenuto alla Redazione il 6 dicembre 2004 e in forma definitiva il 30 giugno 2005. 
Quaternionic maps arise from the higher dimensional gauge theory $(c f .[\mathrm{C}]$, [DT], [FKS], [MS], [NN], [PG]). More precisely they naturally arise from the adiabatic limit of Hermitian Yang-Mills connections on $S U(n)$-bundles on a product of two K3 surfaces. Its linear version in dimension four is the so-called CauchyRiemann-Fueter equation (or quaternionic d-bar equations):

$$
\partial_{x_{1}} f-i \partial_{x_{2}} f-j \partial_{x_{3}} f-k \partial_{x_{4}} f=0
$$

for $f: \mathbb{H} \rightarrow \mathbb{H}$ where $\mathbb{H}$ is the space of quaternions and $x_{1}+i x_{2}+j x_{3}+k x_{4} \in \mathbb{H}$.

Assume $M$ is compact. For any smooth map $u: M \rightarrow N$, consider the energy functional

$$
E(u)=\frac{1}{2} \int_{M}|\nabla u|^{2}
$$

and the functional

$$
E_{T}(u)=A_{\alpha \beta} \int_{M}\left\langle\omega_{J^{\alpha}}, u^{*} \omega_{\mathcal{J}^{\beta}}\right\rangle
$$

and set

$$
I(u)=\frac{1}{2} \int_{M}\left|d u-A_{\alpha \beta} \mathcal{J}^{\beta} \circ d u \circ J^{\alpha}\right|^{2} .
$$

It is clear that $I(u)=0$ if and only if $u$ is a quaternionic map. Since $u$ pulls back the closed 2-form $\omega_{\mathcal{J}^{\beta}}$ to a closed 2-form on $M$ and $\omega_{J^{\alpha}}$ is closed, $E_{T}(u)$ is homotopy invariant and depends on $\left(A_{\alpha \beta}\right)$. The following relation holds $(c f$. [C], [CL1], [FKS])

$$
E(u)+E_{T}(u)=\frac{1}{4} I(u) .
$$

If $u$ is a quaternionic map, then it minimizes energy in its homotopy class so it is harmonic.

Recall [Sc] that a map in the Sobolev space $W^{1,2}(M, N)$ is a stationary harmonic map if it is a critical point of the energy functional with respect to both of the variations on $M$ and $N$ with compact supports. A stationary harmonic map is smooth away from a closed set of zero $(m-2)$-dimensional Hausdorff measure where $m=\operatorname{dim} M$. Let $M$ and $N$ be two hyperkähler manifolds. A map $u$ from $M$ to $N$ is called a stationary quaternionic map if it is a stationary harmonic map and it is a quaternionic map outside its singular set.

It is known that the existence harmonic 2-spheres plays an important role in the study of stationary harmonic maps ([SU], [Lin]).

In this note we investigate the special minimal 2-spheres which arise from the stationary quaternionic maps. We first show that if there are no holomorphic 2spheres in $N$ then any sequence of stationary quaternionic maps with bounded energy subconverges to a stationary quaternionic map strongly in $W^{1,2}(M, N)$. This result was stated and proved in [CL1] when $M$ is of dimension four, and the proof 
we shall present here is essentially based on that in [CL1]. We then find that certain tangent maps of quaternionic maps give rise to an interesting minimal 2-sphere equation:

$$
d f J_{\mathbb{S}^{2}}=-\sum_{k=1}^{3} x_{k} \mathcal{J}^{k} d f
$$

where $f: \mathbb{S}^{2} \rightarrow N,\left(x_{1}, x_{2}, x_{3}\right) \in \mathbb{S}^{2}$ and $J_{\mathbb{S}^{2}}$ is the standard complex structure on $\mathbb{S}^{2}$. We construct a stationary quaternionic map with a codimension-3 singular set by using the embedded minimal $\mathbb{S}^{2}$ in the hyperkähler surface $\widetilde{M}_{2}^{0}$ studied by Atiyah-Hitchin [AH], where $\tilde{M}_{2}^{0}$ is the double cover of the space $M_{2}^{0}$ of centred 2-monopoles on $\mathbb{R}^{3}$ and it is a complete and simply connected hyperkähler surface.

There are interesting results on decomposition of differential forms in quaternionic geometry using representations of special groups (e.g. [Bo], [K], [Sa], [Sw], $[\mathrm{W}]$, etc). It is commented in [W] that the quaternionic maps between hyperkähler manifolds can be described by the splitting of $S p(1)$-representations. The authors thank the referee for his bringing this point and the related references in quaternionic geometry to their attention.

\section{Compactness of stationary quaternionic maps}

A sequence of stationary harmonic maps with bounded energies subconverges to a stationary harmonic map strongly in $W^{1,2}$ topology if there are no harmonic 2spheres in the target manifold [L]. For stationary quaternionic maps, the absence of holomorphic 2-spheres is sufficient to conclude the strong convergence.

Theorem 2.1. Let $M$ and $N$ be compact hyperkähler manifolds with $\operatorname{dim} M=m$. Suppose that $u_{k}$ is a sequence of stationary quaternionic maps with bounded energies. If $N$ does not admit holomorphic $\mathbb{S}^{2}$ 's with respect to the complex structure $a_{i} J^{i}$ on $\mathbb{R}^{2}$ restricted to $\mathbb{S}^{2}$ and the complex structure $a_{i} \mathcal{J}^{i}$ on $N$ for some constants $a_{i}(i=1,2,3)$ with $\sum_{i} a_{i}^{2}=1$, then there is a subsequence of $\left\{u_{k}\right\}$ which converges strongly to a stationary quaternionic map $u$.

Proof. We can always assume that $u_{k} \rightarrow u$ weakly in $W^{1,2}(M, N)$ and that $\left|\nabla u_{k}\right|^{2} d x \rightarrow|\nabla u|^{2} d x+v$ in the sense of measure as $k \rightarrow \infty$. Here $v$ is a nonnegative Radon measure on $M$ with support in $\Sigma$, and $\Sigma$ is the blow-up set of the sequence $u_{k}$ which is $m-2$ rectifiable [L]. We will prove the Hausdorff measure $H^{m-2}(\Sigma)=0$ which implies the strong convergence in $W^{1,2}(M, N)$. Assume $H^{m-2}(\Sigma) \neq 0$. Then [L] there is a nonconstant harmonic map $v: \mathbb{R}^{m} \rightarrow N$ with finite energy and $\nabla_{\Sigma} v=0$. Here we have identified the tangent space of $\Sigma$ at $0 \in \mathbb{R}^{m}=\mathbb{R}^{m-2} \times \mathbb{R}^{2}$ with $\mathbb{R}^{m-2} \times\{0\}$ so $\nabla_{\Sigma}$ means the differentiation along $\mathbb{R}^{m-2} \times\{0\}$. The rescaling process for constructing $v$ is taken place around smooth points of $u_{k}$ which approach 0 , therefore $v$ is also a smooth quaternionic $\operatorname{map}(c f .[\mathrm{CT}])$.

At the point $0 \in \mathbb{R}^{m}$, suppose that $e$ is in the normal direction of $\Sigma$. Let $K$ be the linear space spanned by $J^{\alpha} e$ for $\alpha=1,2,3$, so $K \perp e$. Since rank $d v=2$, we have 
$d v(e) \neq 0$. This implies, from the quaternionic map equation, $\sum_{i=1}^{3} \mathcal{J}^{i} d v\left(J^{i} e\right) \neq$ 0 and in turn $d v\left(J^{i} e\right) \neq 0$ for some $i$. Hence $\operatorname{dim} d v(K)=1$. It then follows that there are real constants $a_{1}, a_{2}, a_{3}$ with $a_{1}^{2}+a_{2}^{2}+a_{3}^{2}=1$ such that $a_{i} J^{i} e \in\{0\} \times \mathbb{R}^{2}$ and $d v\left(a_{i} J^{i} e\right) \neq 0$. Notice that we then have three vectors $a_{i} J^{j} e-a_{j} J^{i} e, i \neq j$ which are perpendicular to $e$ and to $\sum_{i=1}^{3} a_{i} J^{i} e$, so they belong to $T \Sigma$. We therefore have $\left(a_{2} J^{1}-a_{1} J^{2}\right) e \in \operatorname{Ker}(d v),\left(a_{3} J^{1}-a_{1} J^{3}\right) e \in \operatorname{Ker}(d v),\left(a_{2} J^{3}-\right.$ $\left.a_{3} J^{2}\right) e \in \operatorname{Ker}(d v), \mathcal{J}^{\alpha} d v J^{\alpha}=d v$, thus $d v\left(\sum_{i} a_{i} J^{i} e\right)$ can only have components on $\mathcal{J}^{\alpha}(d v(e))$. By a simple calculation, one easily checks that

$$
\begin{aligned}
d v\left(\sum_{i=1}^{3} a_{i} J^{i} e\right)= & \sum_{i, j=1}^{3} \mathcal{J}^{j} d v\left(a_{i} J^{j} J^{i} e\right) \\
= & -\sum_{i=1}^{3} a_{i} \mathcal{J}^{i} d v(e)+\mathcal{J}^{1} d v\left(a_{2} J^{1} J^{2}+a_{3} J^{1} J^{3}\right) e \\
& +\mathcal{J}^{2} d v\left(a_{1} J^{2} J^{1}+a_{3} J^{2} J^{3}\right) e+\mathcal{J}^{3} d v\left(a_{1} J^{3} J^{1}+a_{2} J^{3} J^{2}\right) e \\
= & -\sum_{i=1}^{3} a_{i} \mathcal{J}^{i} d v(e) .
\end{aligned}
$$

At any other point $(0, x)$ in $\mathbb{R}^{m-2} \times \mathbb{R}^{2}$, the vectors $e$ and $\sum_{i=1}^{3} a_{j} \mathcal{J}^{j} e$ still belong to $\{0\} \times \mathbb{R}^{2}$, and the vectors $\left(a_{1} \mathcal{J}^{2}-a_{2} \mathcal{J}^{1}\right) e,\left(a_{2} \mathcal{J}^{2}-a_{3} \mathcal{J}^{2}\right) e,\left(a_{1} \mathcal{J}^{3}-a_{3} \mathcal{J}^{1}\right) e$ lie in $\mathbb{R}^{m-2} \times\{x\}$ hence in the kernel of $d v$ at $(0, x)$, so we can repeat the argument above to conclude $v$ is holomorphic at $(x, 0)$ with respect to the same complex structures $\sum_{i=1}^{3} a_{i} J^{i}$ and $\sum_{i=1}^{3} a_{i} \mathcal{J}^{i}$. It follows that $v$ induces a holomorphic map from $\mathbb{S}^{2}$ to $N$. But no such holomorphic map can exist by assumption. So we must have $H^{m-2}(\Sigma)=0$ and in turn $u_{k}$ converge strongly to $u$ in $W^{1,2}$ norm.

Remark 2.2. The strong convergence is equivalent to $H^{m-2}(\Sigma)=0$ and is equivalent to that the Hausdorff dimension of the singular set $\operatorname{sing}(u)$ of $u$ is no bigger than $m-3$. Moreover $\operatorname{sing}(u)$ is rectifiable since $N$ real analytic [Si].

\section{Quaternionic minimal surfaces via quaternioinc maps}

In this section we study a special class of minimal surfaces which arise from certain tangent maps of the quaternionic maps.

Assume that $M$ is 4-dimensional hyperkähler manifold and $N$ is a $4 n$-dimensional hyperkähler manifold. We can choose a coordinate system around a point $x$ in $M$ so that the matrix expressions of the complex structures on $M$ take the following form:

$$
J^{1}=\left(\begin{array}{cccc}
0 & 0 & 0 & 1 \\
0 & 0 & -1 & 0 \\
0 & 1 & 0 & 0 \\
-1 & 0 & 0 & 0
\end{array}\right), J^{2}=\left(\begin{array}{cccc}
0 & 0 & 1 & 0 \\
0 & 0 & 0 & 1 \\
-1 & 0 & 0 & 0 \\
0 & -1 & 0 & 0
\end{array}\right), J^{3}=\left(\begin{array}{cccc}
0 & -1 & 0 & 0 \\
1 & 0 & 0 & 0 \\
0 & 0 & 0 & 1 \\
0 & 0 & -1 & 0
\end{array}\right)
$$


Note that the three Kähler forms $\omega_{J_{i}}, i=1,2,3$ have variable coefficients in these coordinates. For $f: M \rightarrow N$, if we denote $\frac{\partial f}{\partial x_{k}}$ by $f_{k}$ for $k=1,2,3,4$ in the coordinate system we have just chosen, the quaternionic map equation (1.1) reads

$$
f_{1}-a_{\alpha 3} \mathcal{J}^{\alpha} f_{2}+a_{\alpha 2} \mathcal{J}^{\alpha} f_{3}+a_{\alpha 1} \mathcal{J}^{\alpha} f_{4}=0
$$

where we take summation over $\alpha$.

Now assume that $f$ is a homogeneous degree-0 quaternionic map from $\mathbb{R}^{4}$ to $N$ and satisfies $f\left(x_{1}, x_{2}, x_{3}, x_{4}\right)=f\left(x_{1}, x_{2}, x_{3}, 0\right)$. So $f$ is singular along the $x_{4}$-axis or it is constant. Note that such an $f$ is just a tangent map, with a line of singularities, of a quaternionic map from $M$ to $N$.

As a radially independent harmonic map, $f$ induces a smooth harmonic map from $\mathbb{S}^{2}$ to $N: \phi(x)=f\left(x, x_{4}\right)$ for $x \in \mathbb{S}^{2} \subset \mathbb{R}^{3}$.

Lemma 3.1. With $f$ and $\phi$ as above, then

$$
d \phi J_{\mathbb{S}^{2}}=-a_{\alpha \beta} x_{\beta} \mathcal{J}^{\alpha} d \phi .
$$

Proof. Because $f$ is a homogeneous degree-0 map,

$$
\sum_{k=1}^{4} x_{k} f_{k}=0
$$

and this combined with (3.1) leads to

$$
\left(x_{2}+x_{1} a_{\alpha 3} J^{\alpha}\right) f_{2}+\left(x_{3}-x_{1} a_{\alpha 2} J^{\alpha}\right) f_{3}=0 .
$$

In the spherical coordinates

$$
\left\{\begin{array}{l}
x_{1}=r \sin \alpha \cos \theta \\
x_{2}=r \sin \alpha \sin \theta \\
x_{3}=r \cos \alpha
\end{array}\right.
$$

it reads

$$
\left(x_{2}+x_{1} a_{\alpha 3} J^{\alpha}\right)\left(\cos \alpha \sin \theta f_{\alpha}+\cos \theta \frac{f_{\theta}}{\sin \alpha}\right)+\left(x_{3}-x_{1} a_{\alpha 2} J^{\alpha}\right)\left(-\sin \alpha f_{\alpha}\right)=0 .
$$

Multiplying this equation by $\sin (\alpha)$ yields

$$
\left(x_{2}+x_{1} a_{\alpha 3} J^{\alpha}\right)\left(x_{3} x_{2} f_{\alpha}+x_{1} \frac{f_{\theta}}{\sin \alpha}\right)-\left(x_{3}-x_{1} a_{\alpha 2} J^{\alpha}\right)\left(x_{1}^{2}+x_{2}^{2}\right) f_{\alpha}=0
$$

i.e.

$$
-x_{1}\left(x_{2}+x_{1} a_{\alpha 3} J^{\alpha}\right) \frac{f_{\theta}}{\sin \alpha}=\left(x_{2} x_{3}\left(x_{2}+x_{1} a_{\alpha 3} J^{\alpha}\right)-\left(x_{3}-x_{1} a_{\alpha 2} J^{\alpha}\right)\left(x_{1}^{2}+x_{2}^{2}\right)\right) f_{\alpha} .
$$


Multiplying $x_{2}-x_{1} a_{\alpha 3} J^{\alpha}$ from left on both sides of the equation above, we obtain,

$$
-x_{1}\left(x_{1}^{2}+x_{2}^{2}\right) \frac{f_{\theta}}{\sin \alpha}=x_{1}\left(x_{1}^{2}+x_{2}^{2}\right)\left(x_{1} a_{\alpha 1} J^{\alpha}+x_{2} a_{\alpha 2} J^{\alpha}+x_{3} a_{\alpha 3} J^{\alpha}\right) f_{\alpha}
$$

here we have used $a_{\alpha 3} J^{\alpha} \cdot a_{\beta 2} J^{\beta}=a_{\gamma 1} J^{\gamma}$ with the summation convention over repeated indices applied. So we see $\phi$ satisfies the equation:

$$
d \phi J_{\mathbb{S}^{2}}=-a_{\alpha \beta} x_{\beta} \mathcal{J}^{\alpha} d \phi .
$$

This finishes the proof.

Note that $a_{\alpha \beta} x_{\beta} \mathcal{J}^{\alpha}$ is only defined along the image surface $f\left(\mathbb{S}^{2}\right)$ and $f$ cannot be holomorphic with respect to any complex structure in the 2-sphere family of complex structures on $N$.

Let $\Sigma$ be a Riemann surface, $N^{4 n}$ a hyperkähler manifold with the complex structures $\mathcal{J}^{1}, \mathcal{J}^{2}, \mathcal{J}^{3}$ which satisfy the quaternion relation $\mathcal{J}^{1} \mathcal{J}^{2}=\mathcal{J}^{3}$. Let $\vec{a}=\left(a_{1}, a_{2}, a_{3}\right)$ be smooth functions $\Sigma \rightarrow \mathbb{S}^{2}$.

Definition 3.2. Let $f: \Sigma \rightarrow N^{4 n}$ be a smooth immersion which satisfies

$$
d f J_{\Sigma}=-\sum_{k=1}^{3} a_{k} \mathcal{J}^{k} d f
$$

where $\vec{a}=\left(a_{1}, a_{2}, a_{3}\right): \Sigma \rightarrow \mathbb{S}^{2}$. We say $f$ is a quaternionic surface in $N^{4 n}$. If in addition $f$ is harmonic, we say $f$ is a quaternionic minimal surface.

Condition (3.3) requires the image of $d f$ lying in the span of $\mathcal{J}^{1} d f, \mathcal{J}^{2} d f, \mathcal{J}^{3} d f$. In the twistor space approach to minimal surfaces and harmonic maps, this condition is called "inclusive" (see [AM], [ES], [R], [Sa] and the references therein).

It is not difficult to see that if $f$ satisfies (3.3) then $f$ is conformal. Furthermore, any conformal immersion from $\left(\Sigma, J_{\Sigma}\right)$ to a 4-dimensional hyperkähler manifold satisfies the equation (3.3). In fact, suppose that $e_{1}, e_{2}$ is an orthonormal frame of $\Sigma$. Because $f$ is conformal and $d f\left(e_{1}\right) \perp d f\left(e_{2}\right)$, we have

$$
d f\left(e_{1}\right)=c_{i} J^{i} d f\left(e_{2}\right) \text { and } d f\left(e_{2}\right)=d_{i} J^{i} d f\left(e_{1}\right)
$$

with $\sum_{i} c_{i}^{2}=1$ and $\sum_{i} d_{i}^{2}=1$. It is clear that

$$
c_{i}\left|d f\left(e_{2}\right)\right|^{2}=\left\langle d f\left(e_{1}\right), J^{i} d f\left(e_{2}\right)\right\rangle=-\left\langle J^{i} d f\left(e_{1}\right), d f\left(e_{2}\right)\right\rangle=-d_{i}\left|d f\left(e_{1}\right)\right|^{2} .
$$

Since $\left|d f\left(e_{2}\right)\right|^{2}=\left|d f\left(e_{1}\right)\right|^{2}=1 / 2|d f|^{2}$, we have $c_{i}=-d_{i}$ hence (3.3) holds.

Lemma 3.3. Let $u: \Sigma_{1} \rightarrow \Sigma_{2}$ be a holomorphic map between two Riemann surfaces with complex structures $J_{\Sigma_{1}}$ and $J_{\Sigma_{2}}$ respectively. Then for any smooth map $f: \Sigma_{2} \rightarrow N$ which satisfies (3.3) with $\vec{a}: \Sigma_{1} \rightarrow \mathbb{S}^{2}, f \circ u: \Sigma_{1} \rightarrow N$ satisfies (3.3) with $\vec{a} \circ u: \Sigma_{1} \rightarrow \mathbb{S}^{2}$. If $f\left(\Sigma_{2}\right)$ is a quaternionic minimal surface, then $f \circ u\left(\Sigma_{1}\right)$ is also a quaternionic minimal surface. 
Proof. Then for any $x \in \Sigma_{1}$

$$
\begin{aligned}
d(f \circ u)_{x} J_{\Sigma_{1}}(x) & =d f_{u(x)} \circ d u_{x} J_{\Sigma_{1}}(x) \\
& =d f_{u(x)} \circ J_{\Sigma_{2}}(u(x)) d u_{x} \\
& =-a_{i}(u(x)) \mathcal{J}_{u(x)}^{i} d f_{u(x)} \circ d u_{x} \\
& =-a_{i}(u(x)) \mathcal{J}_{u(x)}^{i} d(f \circ u)_{x} .
\end{aligned}
$$

If $f$ is harmonic and $u$ is holomorphic, $f \circ u$ is harmonic.

Proposition 3.4. A quaternionic surface in $N^{4 n}$ is a minimal surface if and only if $\vec{a}$ is holomorphic with respect to the complex structure on $\Sigma$ which makes the metric $g$ Hermitian and the standard complex structure on $\mathbb{S}^{2} . \vec{a}$ is constant if and only if the quaternionic surface is a holomorphic curve.

Proof. Since $f$ is conformal, a quaternionic surface in $N^{4 n}$ is a minimal surface if and only if $f$ is a harmonic map from $\Sigma$ to $N$. Let $e_{1}, e_{2}$ be an orthonormal frame on $\Sigma$ which satisfies $I e_{1}=e_{2}, I e_{2}=-e_{1}$. Note that, by the definition,

$$
f_{1}:=d f\left(e_{1}\right)=\sum_{i=1}^{3} a_{i} J^{i} f_{2}, \quad f_{2}:=d f\left(e_{2}\right)=-\sum_{i=1}^{3} a_{i} J^{i} f_{1} .
$$

Taking the normal coordinates centred at $x$ and $f(x)$, we have

$$
\begin{aligned}
\triangle f= & -\nabla_{2}\left(\sum_{i=1}^{3} a_{i} J^{i}\right) f_{1}+\nabla_{1}\left(\sum_{i=1}^{3} a_{i} J^{i}\right) f_{2} \\
= & \left(-\sum_{i=1}^{3} \nabla_{2} a_{i} J^{i}-\left(\sum_{i=1}^{3} \nabla_{1} a_{i} J^{i}\right)\left(\sum_{i=1}^{3} a_{i} J^{i}\right)\right) f_{1} \\
= & \left(-\nabla_{2} a_{1}-a_{3} \nabla_{1} a_{2}+a_{2} \nabla_{1} a_{3}\right) J^{1} f_{1} \\
& +\left(-\nabla_{2} a_{2}-a_{1} \nabla_{1} a_{3}+a_{3} \nabla_{1} a_{1}\right) J^{2} f_{1} \\
& +\left(-\nabla_{2} a_{3}-a_{2} \nabla_{1} a_{1}+a_{1} \nabla_{1} a_{2}\right) J^{3} f_{1} .
\end{aligned}
$$

Since $f$ is harmonic, it follows that

$$
\left\{\begin{array}{l}
\nabla_{2} a_{1}+a_{3} \nabla_{1} a_{2}-a_{2} \nabla_{1} a_{3}=0 \\
\nabla_{2} a_{2}+a_{1} \nabla_{1} a_{3}-a_{3} \nabla_{1} a_{1}=0 \\
\nabla_{2} a_{3}+a_{2} \nabla_{1} a_{1}-a_{1} \nabla_{1} a_{2}=0 .
\end{array}\right.
$$

Solving (3.5) and using $a_{1} \nabla_{2} a_{1}+a_{2} \nabla_{2} a_{2}+a_{3} \nabla_{2} a_{3}=0$, one gets

$$
\left\{\begin{array}{l}
\nabla_{1} a_{1}+a_{2} \nabla_{2} a_{3}-a_{3} \nabla_{2} a_{2}=0 \\
\nabla_{1} a_{2}+a_{3} \nabla_{2} a_{1}-a_{1} \nabla_{2} a_{3}=0 \\
\nabla_{1} a_{3}+a_{1} \nabla_{2} a_{2}-a_{2} \nabla_{2} a_{1}=0 .
\end{array}\right.
$$


We can rewrite (3.5) as

$$
\nabla_{2} \vec{a}=\vec{a} \times \nabla_{1} \vec{a}
$$

and rewrite (3.6) as

$$
\nabla_{1} \vec{a}=-\vec{a} \times \nabla_{2} \vec{a}
$$

Noting that the standard complex structure on $\mathbb{S}^{2}$ at $\vec{a}$ is $\vec{a} \times$, we can see that $\vec{a}$ satisfies the equations (3.5) and (3.6) if and only if it is a holomorphic map with respect to the complex structure on $\Sigma$ which makes the metric $g$ Hermitian and the standard complex structure on $\mathbb{S}^{2}$.

Remark that if we write the equation in $b_{i}=-a_{i}$ then $\vec{b}$ is anti-holomorphic and if $N$ is 4-dimensional the above result was obtained in [ES] and by S.S. Chern if $N=\mathbb{R}^{4}$.

In particular, when a quaternionic surface is minimal, the mapping $\vec{a}$ satisfies the harmonic map equation to the standard sphere:

$$
\Delta \vec{a}+|\nabla \vec{a}|^{2} \vec{a}=0 .
$$

The following theorem is known to be true for minimal surface in a Kähler-Einstein manifold of real dimension 4 (cf. [CW]) by noticing that $a_{k}=\cos \alpha_{k}$ where $\alpha_{k}$ is the Kähler angle of the surface $f(\Sigma)$ with respect to the Kähler form $\omega_{\mathcal{J}^{k}}$ in $N$.

Theorem 3.5. If a quaternionic surface in $N^{4 n}$ is a minimal surface with $\vec{a}=$ $\left(a_{1}, a_{2}, a_{3}\right): \Sigma \rightarrow \mathbb{S}^{2}$, then

$$
\Delta a_{k}+2 \frac{\left|\nabla a_{k}\right|^{2} a_{k}}{1-a_{k}^{2}}=0 .
$$

Proof. We only need to prove the result for $a_{1}$. First we compute the Laplacian of $a_{1}$ as follows. Again we take the normal coordinates centred at $x \in M$ and at $f(x) \in N$. Differentiating in $\nabla_{2}$ of

$$
\nabla_{2} a_{1}=a_{2} \nabla_{1} a_{3}-a_{3} \nabla_{1} a_{2}
$$

yields

$$
\nabla_{22}^{2} a_{1}=\nabla_{2} a_{2} \nabla_{1} a_{3}+a_{2} \nabla_{12}^{2} a_{3}-\nabla_{2} a_{3} \nabla_{1} a_{2}-a_{3} \nabla_{12}^{2} a_{2} .
$$

Multiplying $a_{3}, a_{2}, a_{1}$ accordingly to the following three equations

$$
\begin{aligned}
& a_{3} \nabla_{1} a_{1}=\nabla_{2} a_{2}+a_{1} \nabla_{1} a_{3} \\
& a_{2} \nabla_{1} a_{1}=a_{1} \nabla_{1} a_{2}-\nabla_{2} a_{3} \\
& a_{1} \nabla_{1} a_{1}=-a_{2} \nabla_{1} a_{2}-a_{3} \nabla_{1} a_{3}
\end{aligned}
$$

then summing them up leads to

$$
\nabla_{1} a_{1}=a_{3} \nabla_{2} a_{2}-a_{2} \nabla_{2} a_{3}
$$


Differentiating in $\nabla_{1}$ gives

$$
\nabla_{11}^{2} a_{1}=\nabla_{1} a_{3} \nabla_{2} a_{2}+a_{3} \nabla_{21}^{2} a_{2}-\nabla_{1} a_{2} \nabla_{2} a_{3}-a_{2} \nabla_{21}^{2} a_{3} .
$$

Now we conclude

$$
\Delta a_{1}=2\left(\nabla_{1} a_{3} \nabla_{2} a_{2}-\nabla_{1} a_{2} \nabla_{2} a_{3}\right)
$$

and we may write the right hand side in terms which only involve $\nabla_{1}$ as follows:

$$
\begin{aligned}
\nabla_{1} a_{3} \nabla_{2} a_{2}-\nabla_{1} a_{2} \nabla_{2} a_{3}= & \nabla_{1} a_{3}\left(a_{3} \nabla_{1} a_{1}-a_{1} \nabla_{1} a_{3}\right) \\
& -\nabla_{1} a_{2}\left(a_{1} \nabla_{1} a_{2}-a_{2} \nabla_{1} a_{1}\right) \\
= & a_{3} \nabla_{1} a_{1} \nabla_{1} a_{3}-a_{1}\left|\nabla_{1} a_{3}\right|^{2} \\
& -a_{1}\left|\nabla_{1} a_{2}\right|^{2}+a_{2} \nabla_{1} a_{1} \nabla_{1} a_{2} \\
= & -a_{1}\left(\left|\nabla_{1} a_{1}\right|^{2}+\left|\nabla_{1} a_{2}\right|^{2}+\left|\nabla_{1} a_{3}\right|^{2}\right) .
\end{aligned}
$$

So we have just shown

$$
\Delta a_{1}=-2 a_{1}\left(\left|\nabla_{1} a_{1}\right|^{2}+\left|\nabla_{1} a_{2}\right|^{2}+\left|\nabla_{1} a_{3}\right|^{2}\right) .
$$

On the other hand, we have

$$
\begin{aligned}
\left|\nabla a_{1}\right|^{2} & =\left|\nabla_{1} a_{1}\right|^{2}+\left|\nabla_{2} a_{1}\right|^{2} \\
& =\left|\nabla_{1} a_{1}\right|^{2}+\left(a_{2} \nabla_{1} a_{3}-a_{3} \nabla_{1} a_{2}\right)^{2} \\
& =\left|\nabla_{1} a_{1}\right|^{2}+a_{2}^{2}\left|\nabla_{1} a_{3}\right|^{2}+a_{3}^{2}\left|\nabla_{1} a_{2}\right|^{2}-2 a_{2} a_{3} \nabla_{1} a_{2} \nabla_{1} a_{3} .
\end{aligned}
$$

However,

$$
\begin{aligned}
& \left(1-a_{1}^{2}\right)\left(\left|\nabla_{1} a_{1}\right|^{2}+\left|\nabla_{1} a_{2}\right|^{2}+\left|\nabla_{1} a_{3}\right|^{2}\right)-\left|\nabla a_{1}\right|^{2} \\
= & -a_{1}^{2}\left|\nabla_{1} a_{1}\right|^{2}+\left(1-a_{1}^{2}-a_{3}^{2}\right)\left|\nabla_{1} a_{2}\right|^{2}+\left(1-a_{1}^{2}-a_{2}^{2}\right)\left|\nabla_{1} a_{3}\right|^{2}+2 a_{2} a_{3} \nabla_{1} a_{2} \nabla_{1} a_{3} \\
= & -a_{1}^{2}\left|\nabla_{1} a_{1}\right|^{2}+a_{2}^{2}\left|\nabla_{1} a_{2}\right|^{2}+a_{3}^{2}\left|\nabla_{1} a_{3}\right|^{2}+2 a_{2} a_{3} \nabla_{1} a_{2} \nabla_{1} a_{3} \\
= & 0
\end{aligned}
$$

by recalling $a_{1} \nabla_{1} a_{1}=a_{2} \nabla_{1} a_{2}+a_{3} \nabla_{1} a_{3}$.

Putting (3.8) and (3.9) together, we have

$$
\Delta a_{1}=-2 \frac{\left|\nabla a_{1}\right|^{2} a_{1}}{1-a_{1}^{2}},
$$

which completes the proof.

Theorem 3.6. Suppose that $f$ is a minimal quaternionic surface in $N^{4}$. Then either $f$ is constant or the Euler characteristic number $\frac{1}{2 \pi} \chi(N f(\Sigma))$ of the normal bundle of $f(\Sigma)$ is $2 g-2-2 \operatorname{deg} \vec{a}$. In particular, if $f \in C^{2}\left(\mathbb{S}^{2}, N^{4}\right)$ satisfies the equation

$$
d f J_{\mathbb{S}^{2}}=-\sum_{i=1}^{3} x_{i} \mathcal{J}^{i} d f,
$$

where $x \in \mathbb{S}^{2} \subset \mathbb{R}^{3}$, then either $f$ is constant or the Euler characteristic number of the normal bundle of $f\left(\mathbb{S}^{2}\right)$ is -4 . 
Proof. Let $\Sigma_{0}=f(\Sigma) . \quad \Sigma_{0}$ is a minimal surface in $N$ because $f$ is harmonic and conformal. Proposition 4.2 and Proposition 4.3 in [CT] assert, for a compact minimal surface in a Kähler-Einstein surface $N$, that the generalized adjunction formula

$$
\begin{aligned}
\chi\left(T \Sigma_{0}\right)+\chi\left(N \Sigma_{0}\right) & =\int_{\Sigma} \Omega_{12}+\Omega_{34}-\frac{1}{2} \int_{\Sigma}\left|\nabla J_{\Sigma_{0}}\right|^{2} \\
& =2 \pi \int_{\Sigma} \alpha c_{1}(N)-\frac{1}{2} \int_{\Sigma}\left|\nabla J_{\Sigma}\right|^{2}
\end{aligned}
$$

holds for some function $\alpha$ on $\Sigma_{0}$, where $\Omega_{12}, \Omega_{34}$ are the curvature tensors of $N$ along the tangential and normal directions of $\Sigma_{0}$ respectively. The term $\left|\nabla J_{\Sigma_{0}}\right|^{2}$ is equal to $2\left|h_{12}^{4}-h_{11}^{3}\right|^{2}+2\left|h_{22}^{4}-h_{12}^{3}\right|^{2}$ where $h_{i j}^{k}$ are the second fundamental forms of $\Sigma_{0}$ in $N$.

Since $c_{1}(N)=0$, we have

$$
\chi\left(T \Sigma_{0}\right)+\chi\left(N \Sigma_{0}\right)=-\frac{1}{2} \int_{\Sigma_{0}}\left|\nabla J_{\Sigma_{0}}\right|^{2} .
$$

In particular, an embedded holomorphic $\mathbb{S}^{2}$ has self-intersection number -2 in $M$ with $C_{1}(M)=0$.

On the other hand, for any solution of (3.5), by Proposition 3.4 and Theorem 3.5 and Proposition 3.2 in [CL2] (specializing the general formula for cosine of the Kähler angle along the mean curvature flow to minimal surface) and (3.7), we always have

$$
\left|\nabla J_{\Sigma_{0}}\right|^{2}=|\nabla \vec{a}|^{2}=\frac{2\left|\nabla a_{i}\right|^{2}}{1-a_{i}^{2}}
$$

for $i=1,2,3$. One then has

$$
\begin{aligned}
\frac{1}{2 \pi} \chi(N \Sigma) & =-\frac{1}{4 \pi} \int_{\Sigma_{g}}|\nabla \vec{a}|^{2}+2 g-2 \\
& =2 g-2-2 \operatorname{deg} \vec{a} .
\end{aligned}
$$

Here we recall for holomorphic $\vec{a}$ to $\mathbb{S}^{2}$,

$$
\operatorname{deg} \vec{a}=\frac{1}{\operatorname{vol}\left(\mathbb{S}^{2}\right)} \int_{\Sigma_{g}} \operatorname{Jac}(\vec{a})=\frac{1}{4 \pi} \int_{\Sigma_{g}}|\partial \vec{a}|^{2}=\frac{1}{8 \pi} \int_{\Sigma_{g}}|\nabla \vec{a}|^{2} .
$$

Now if $\Sigma=\mathbb{S}^{2}$ and $\vec{a}(x)=\left(x_{1}, x_{2}, x_{3}\right), f: \mathbb{S}^{2} \rightarrow N$ is harmonic because $\vec{a}: \mathbb{S}^{2} \rightarrow \mathbb{S}^{2}$ is the identity map. We conclude

$$
\frac{1}{2 \pi} \chi(N \Sigma)=-2-\frac{1}{4 \pi} \int_{\mathbb{S}^{2}}|\nabla x|^{2}=-4 .
$$

This completes the proof. 
Based on the results we obtained so far, we next construct an example of stationary quaternionic map from $\mathbb{R}^{4}$ with a line of singularities. For any smooth map $\phi: \mathbb{S}^{2} \rightarrow N$, we have an extension $f\left(x, x_{4}\right):=\phi(x /|x|)$ for any $x \in \mathbb{R}^{3} \backslash\{0\}$. Moreover, the proof of Lemma 3.1 can be reversed to produce a quaternionic map with the $x_{4}$-axis as its singular set from a map $\phi$ which satisfies (3.2).

In the monograph $[\mathrm{AH}]$, Atiyha and Hitchin considered the space $M_{2}^{0}$ of centred 2-monopoles on $\mathbb{R}^{3}$ with finite action. It is a complete hyperkähler manifold of dimension 4. $S O(3)$ acts on $M_{2}^{0}$ isometrically and this action lifts to a double (also Riemannian universal) covering $\widetilde{M}_{2}^{0}$. The space of axisymmetric monopoles, which constitute a special class of solutions to the monopole equations, defines an embedded minimal $\mathbb{R} P^{2}$ in $M_{2}^{0}$. This $\mathbb{R} P^{2}$ lifts to an embedded minimal $\mathbb{S}^{2}$ in the hyperkähler manifold $\widetilde{M}_{2}^{0}$.

Corollary 3.7. There does exist a nontrivial minimal quaternionic sphere $\phi$ in the hyperkähler manifold $\widetilde{M}_{2}^{0}$ with $\vec{a}=\left(x_{1}, x_{2}, x_{3}\right)$. The extended map $f$ from $\phi$ is a stationary quaternionic map from $\mathbb{R}^{4}$ to $\widetilde{M}_{2}^{0}$ with the entire $x_{4}$-axis as singular set.

Proof. We take the nontrivial embedded minimal $\mathbb{S}^{2}$ in $\widetilde{M}_{2}^{0}$ discussed above. The Euler characteristic number of the normal bundle of this minimal 2-sphere is -4 as shown in $[\mathrm{AH}]$.

By Theorem 3.6, we know that the minimal 2-sphere is a minimal quaternionic sphere $\phi_{0}$ with a function $\vec{a}_{0}$ in its definition, and deg $\vec{a}_{0}=1$. Since $\vec{a}_{0}: \mathbb{S}^{2} \rightarrow \mathbb{S}^{2}$ is holomorphic and of degree 1 , it is diffeomorphic because the sum of orders of the zeros of $\left|\partial \vec{a}_{0}\right|$ is $-\operatorname{deg}\left(\vec{a}_{0}\right)(2 \cdot 0-2)+(2 \cdot 0-2)=0,\left|\partial \vec{a}_{0}\right|$ has no zeros, and therefore the inverse $\vec{a}_{0}^{-1}$ of $\vec{a}_{0}$ exists and is holomorphic. So, $\phi:=\phi_{0} \circ \vec{a}_{0}^{-1}$ is a nontrivial minimal quaternionic sphere with $\vec{a}=\left(x_{1}, x_{2}, x_{3}\right)$ by Lemma 3.3.

Recall that action of the complex structure $J_{\mathbb{S}^{2}}$ at $x \in \mathbb{S}^{2}$ is given by the standard cross product $x \times$. Write $\vec{a}_{0}=\left(a_{01}, a_{02}, a_{03}\right)$. Then

$$
a_{0 i}(x)=-\frac{\left\langle d \phi_{0}(x \times e), \mathcal{J}^{i} d \phi_{0}(e)\right\rangle_{x}}{\left|d \phi_{0 x}(e)\right|^{2}}
$$

and $d \phi_{0}$ at $x$ is the same as $d \phi$ at $-x$ because $\phi_{0}$ is the lift from $\mathbb{R} P^{2}$. We then conclude

$$
\vec{a}_{0}(-x)=-\vec{a}_{0}(x), \quad \vec{a}_{0}^{-1}(-x)=-\vec{a}_{0}^{-1}(x) .
$$

The chain rule implies

$$
\begin{aligned}
|\nabla \phi(-x)|^{2} & =\left|\nabla \phi_{0}\left(\vec{a}_{0}^{-1}(-x)\right)\right|^{2}\left|\nabla \vec{a}_{0}^{-1}(-x)\right|^{2} \\
& =\left|\nabla \phi_{0}\left(-\vec{a}_{0}^{-1}(x)\right)\right|^{2}\left|-\nabla \vec{a}_{0}^{-1}(x)\right|^{2} \\
& =\left|\nabla \phi_{0}\left(\vec{a}_{0}^{-1}(x)\right)\right|^{2}\left|\nabla \vec{a}_{0}^{-1}(x)\right|^{2}=|\nabla \phi(x)|^{2}
\end{aligned}
$$


because $\phi_{0}$ is the lift from $\mathbb{R} P^{2}$. Therefore for $i=1,2,3$,

$$
\int_{\mathbb{S}^{2}} x_{i}|\nabla \phi|^{2}=0
$$

The fact that the extended map $f$ is stationary follows from the lemma below.

The lemma below is known to experts. For the sake of completeness, we present a proof of it.

Lemma 3.8. Let $\phi$ be a smooth harmonic map from $\mathbb{S}^{2}$ to a Riemannian manifold $N$. Then the extended map $f$ of $\phi$, which is defined by $f\left(x, x^{\prime}\right)=\phi(x /|x|)$ for $x=\left(x_{1}, x_{2}, x_{3}\right) \neq(0,0,0), x^{\prime} \in\{0\} \times \mathbb{R}^{m-3} \subset \mathbb{R}^{m}$, is a stationary harmonic map if and only if $\phi$ satisfies

$$
\int_{\mathbb{S}^{2}} x_{i}|\nabla \phi|^{2}=0, \quad i=1,2,3, \quad\left(x_{1}, x_{2}, x_{3}\right) \in \mathbb{S}^{2} .
$$

Proof. In fact, we have

$$
\nabla_{x^{\prime}} f=0, \frac{\partial f}{\partial r}=0, r=\sqrt{x_{1}^{2}+x_{2}^{2}+x_{3}^{2}} .
$$

Define a cut-off function by

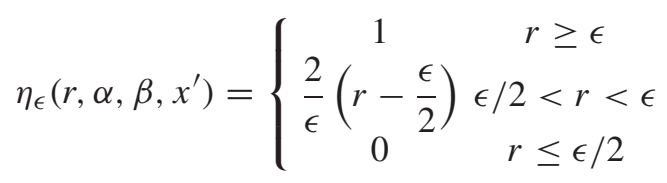

where $x_{1}=r \sin \alpha \cos \beta, x_{2}=r \sin \alpha \sin \beta, x_{3}=r \cos \beta$.

For any smooth vector field $X=\left(X_{1}, \cdots, X_{m}\right)$ in $\mathbb{R}^{m}$ with compact support, because $f$ is smooth away from $\{0\} \times \mathbb{R}^{m-3}$, we have

$$
\begin{aligned}
0= & \int_{\mathbb{R}^{m}}\left(|\nabla f|^{2} \delta_{i j}-2 \nabla_{i} f \nabla_{j} f\right) \nabla_{j}\left(\eta_{\epsilon} X_{i}\right) \\
= & \int_{\mathbb{R}^{m}}\left(|\nabla f|^{2} \delta_{i j}-2 \nabla_{i} f \nabla_{j} f\right) \nabla_{j} \eta_{\epsilon} X_{i} \\
& +\int_{\mathbb{R}^{m}}\left(|\nabla f|^{2} \delta_{i j}-2 \nabla_{i} f \nabla_{j} f\right) \eta_{\epsilon} \nabla_{j} X_{i} .
\end{aligned}
$$

It then follows

$$
\begin{aligned}
\int_{\mathbb{R}^{m}}\left(|\nabla f|^{2} \delta_{i j}-2 \nabla_{i} f \nabla_{j} f\right) \nabla_{j} X_{i} & =\lim _{\epsilon \rightarrow 0} \int_{\mathbb{R}^{m}}\left(|\nabla f|^{2} \delta_{i j}-2 \nabla_{i} f \nabla_{j} f\right) \eta_{\epsilon} \nabla_{j} X_{i} \\
& =-\lim _{\epsilon \rightarrow 0} \int_{\mathbb{R}^{m}}\left(|\nabla f|^{2} \delta_{i j}-2 \nabla_{i} f \nabla_{j} f\right) \nabla_{j} \eta_{\epsilon} X_{i}
\end{aligned}
$$


Therefore, $f$ is stationary if and only if

$$
\lim _{\epsilon \rightarrow 0} \int_{\mathbb{R}^{m}}\left(|\nabla f|^{2} \delta_{i j}-2 \nabla_{i} f \nabla_{j} f\right) \nabla_{j} \eta_{\epsilon} X_{i}=0 .
$$

Direct computation shows that the above condition is equivalent to

$$
\int_{\mathbb{R}^{m-3}} \int_{\mathbb{S}^{2}}|\nabla \phi|^{2} \sum_{i=1}^{3} x_{i} X_{i}\left(0, x^{\prime}\right) d \sigma d x^{\prime}=0 .
$$

Since $X$ is arbitrary, we see the desired statement holds.

\section{References}

[1] D. V. Alekseevsky and S. MarchiafaVa, A twistor construction of Kähler submanifolds of a quaternionic Kähler manifold, Ann. Mat. Pura Appl. 184 (2005), 53-74.

[2] D. Anselmi and P. FrÉ, Topological Sigma-Models in Four Dimensions and Triholomorphic Maps, Nucl. Phys. B416 (1994), 255-300.

[3] M. AtiYah and N. Hitchin, "The geometry and dynamics of magnetic monopoles", Princeton University Press 1988.

[4] F. Bethuel, On the singular set of stationary harmonic maps, Manuscripta Math. 78 (1993), 417-443.

[5] J. CHEN, Complex anti-self-dual connections on product of Calabi-Yau surfaces and triholomorphic curves, Comm. Math. Phys. 201 (1999), 201-247.

[6] J. CHEN and J. Li, Quaternionic maps between hyperkähler manifolds, J. Differential Geom. 55 (2000), 355-384.

[7] J. CHEN and J. LI, Mean curvature flow of surface in 4-manifolds, Adv. Math. 163 (2001), 287-309.

[8] J. Chen and G. Tian, Minimal surfaces in Riemannian 4-manifolds, Geom. Funct. Anal. 7 (1997), 873-916.

[9] S. S. CHERn and J. Wolfson, Minimal surfaces by moving frames, Amer. J. Math. 105 (1983), 59-83.

[10] S. Donaldson and R. Thomas, Gauge theory in higher dimensions, In: "The Geometric Universe: Science, Geometry and the work of Roger Penrose", S. A. Huggett et al. (eds), Oxford Univ. Press, 1998, pp. 31-47.

[11] L. C. Evens, Partial regularity for stationary harmonic maps, Arch. Rat. Mech. Anal. 116 (1991), 101-112.

[12] J. Eells and S. SAlAmon, Twistorial construction of harmonic maps of surfaces into four-manifolds, Ann. Scuola Norm. Sup. Pisa Cl. Sci. (4) 12 (1985), 589-640.

[13] J. M. Figuroa-O'FArrill, C. KöHL and B. Spence, Supersymmetric Yang-Mills, octonionic instantons and triholomorphic curves, Nucl. Phys. B521 (1998), 419-443.

[14] D. JoYCE, Hypercomplex algebraic geometry, Quart. J. Math. 49 (1998), 129-162.

[15] F. H. LIN, Gradient estimates and blow-up analysis for stationary harmonic maps, Ann. Math. 149 (1999), 785-829.

[16] J. Li and G. TIAn, A blow-up formula for stationary harmonic maps, Internat. Math. Res. Notices 14 (1998), 735-755.

[17] C. Mamone and S. SAlamon, Yang-Mills fields on quaternionic spaces. Nonlinearity 1 (1988), 517-530. 
[18] Y. NAGATOMO and T. NitTA, $k$-instantons on $G_{2}\left(C^{n+2}\right)$ and stable vector bundles. Math. Z. 232 (1999), 721-737.

[19] Y. S. Poon and K. GALICKI, Duality and Yang-Mills fields on quaternionic Kähler manifolds. J. Math. Phys. 32 (1991), 1263-1268.

[20] J. RAWNSLEY, f-structures, f-twistor spaces and harmonic maps, In: "Geometry Seminar 'Luigi Bianchi', II - 1984”, E. Vesentini (ed.), Lect. Nothes Math. 1164, Springer, Berlin, 1985, 85-159.

[21] S. Salamon, Harmonic and holomorphic maps, In: "Geometry Seminar 'Luigi Bianchi', II - 1984”, E. Vesentini (ed.), Lect. Notes Math. 1164, Springer, Berlin, 1985, 161-224.

[22] R. Schoen, Analytic aspects of harmonic maps, In: "Seminar on nonlinear Partial Differential equations", S. S. Chern (ed.), M.S.R.I. Publications 2, Springer-Verlag, NewYork, 1984, 321-358.

[23] L. Simon, Rectifiability of the singular set of energy minimizing maps, Calc. Var. Partial Differential Equations 3 (1995), 1-65.

[24] A. Swann, Quaternionic Kähler Geometry and the Fundamental 4-form, In: "Proc. Curvature Geom. workshop", C. T. J. Dodson (ed.), ULDM Publications Lancaster, 1989, $165-173$.

[25] J. SACKS and K. UHLENBECK, The existence of minimal immersions of 2-spheres, Ann. of Math. (2) 113 (1981), 1-24.

[26] D. WidDows, A Dolbeault-type double complex on quaternionic manifolds, Asian J. Math. 6 (2002), 253-275.

Department of Mathematics

The University of British Columbia Vancouver, BC, Canada V6T 1Z2

jychen@math.ubc.ca

Math. Group

The abdus salam ICTP

Trieste 34100 Italy

and

Academy of Mathematics

and Systems Sciences

Chinese Academy of Sciences

Beijing 100080, P. R. of China.

jyli@ictp.it 